\title{
Adult-Onset Hyperplasia of An Ectopic Cervical Thymus Associated with Uncontrolled Hyperthyroidism: A Case Report
}

\author{
Hiroaki Takahashi, ${ }^{1,2,}$ Manabu Minami, ${ }^{1}$ Tomohiko Masumoto, ${ }^{1}$ Takashi Hiyama, ${ }^{1}$ Yuka Kujiraoka, ${ }^{3}$ \\ Mariko Nakamagoe, ${ }^{4}$ and Yuko Minami ${ }^{5,6}$ \\ ${ }^{1}$ Department of Diagnostic Radiology, University of Tsukuba Hospital, Ibaraki, Japan \\ ${ }^{2}$ Department of Radiology, Ibaraki Prefectural Central Hospital, Ibaraki, Japan \\ ${ }^{3}$ Department of Diagnostic Radiology, Tsukuba Memorial Hospital, Ibaraki, Japan \\ ${ }^{4}$ Department of Otolaryngology, University of Tsukuba Hospital, Ibaraki, Japan \\ ${ }^{5}$ Department of Pathology, University of Tsukuba Hospital, Ibaraki, Japan \\ ${ }^{6}$ Chest Disease and Disabilities, National Hospital Organization Ibarakihigashi National Hospital, Ibaraki, Japan \\ "Corresponding author: Hiroaki Takahashi, Department of Diagnostic Radiology, University of Tsukuba Hospital, Ibaraki, Japan, E-mail: h.1982.takahashi@gmail.com
}

Received 2016 May 28; Revised 2016 June 20; Accepted 2016 June 30.

\begin{abstract}
Ectopic cervical thymus (ECT) is an essentially benign condition that is identified during the development of the thymus, and is rare among the adult population. Thymic hyperplasia is another thymus-associated condition, which is known to occur secondary to hyperthyroidism. A 39-year-old woman with hyperthyroidism presented with an enlarging submandibular mass, which was diagnosed as a benign hyperplasia of the ECT. Computed tomography and magnetic resonance imaging largely contributed to its diagnosis, and an unnecessary surgical resection was avoided. To the best of our knowledge, this is the first report that describes clinically significant hyperthyroidism-induced ECT hyperplasia during adulthood.
\end{abstract}

Keywords: Thymic Hyperplasia, Hyperthyroidism, Chemical Shift Imaging, Ectopic Thymus, Magnetic Resonance Imaging, Computed Tomography

\section{Introduction}

An ectopic cervical thymus (ECT) is an essentially benign anomaly that results from thymic descent failure, and occurs mostly in the prepubertal pediatric population (13). In adults, cases of ECT are rare, most likely because of age-related involution and replacement by fibroadipose tissue (1-4). Thymic hyperplasia is another medical condition associated with thymic tissue, and is known to occur following hyperthyroidism and regress after appropriate anti-thyroid therapy (5-7). However, it remains unknown whether hyperthyroidism may cause clinically significant ECT hyperplasia during adulthood.

Here, we report the unusual case of a 39-year-old woman with uncontrolled hyperthyroidism who presented with an enlarging submandibular mass, which was diagnosed as a benign ECT hyperplasia.

\section{Case Presentation}

A 39-year-old woman with an enlarging soft mass on the right side of the neck visited our hospital. The woman reported neck discomfort but no neck pain. When first recognized nearly 10 years prior to the woman's visit, the mass was much smaller, as well as constant in size. However, 2 months prior to her visit, the patient noticed the mass had grown in size and this made her feel uncomfortable. On physical examination, the mass was located in the right submandibular area. The overlying skin was normal and no tenderness was detected on palpation.

Computed tomography (CT) study demonstrated a well-defined, homogeneous mass with weak contrast enhancement, which was located between the right submandibular gland and carotid artery (Figure 1A). The thyroid gland appeared heterogeneous and moderately enlarged, suggesting thyroid abnormality (Figure 1B). The normally positioned thymus was also identified in the anterior mediastinum and appeared enlarged (Figure 1C). Magnetic resonance (MR) T2-weighted images (T2WI) revealed the mass as homogeneously hyperintense (Figure 1D), while chemical shift T1-weighted images (T1WI) showed slight signal loss on out-of-phase images compared to in-phase images, suggesting the presence of microscopic fat (Figure $1 \mathrm{G}$ and $1 \mathrm{H}$ ). Diffusion-weighted images (DWI) showed restricted diffusion in the mass (Figure $1 \mathrm{E})$, and contrast-enhanced T1WI showed homogeneously weak enhancement (Figure 1F). The mass did not contain calcification or necrotic tissue.

Aside from the submandibular mass, the patient revealed a 3-month history of Graves' disease with wors- 

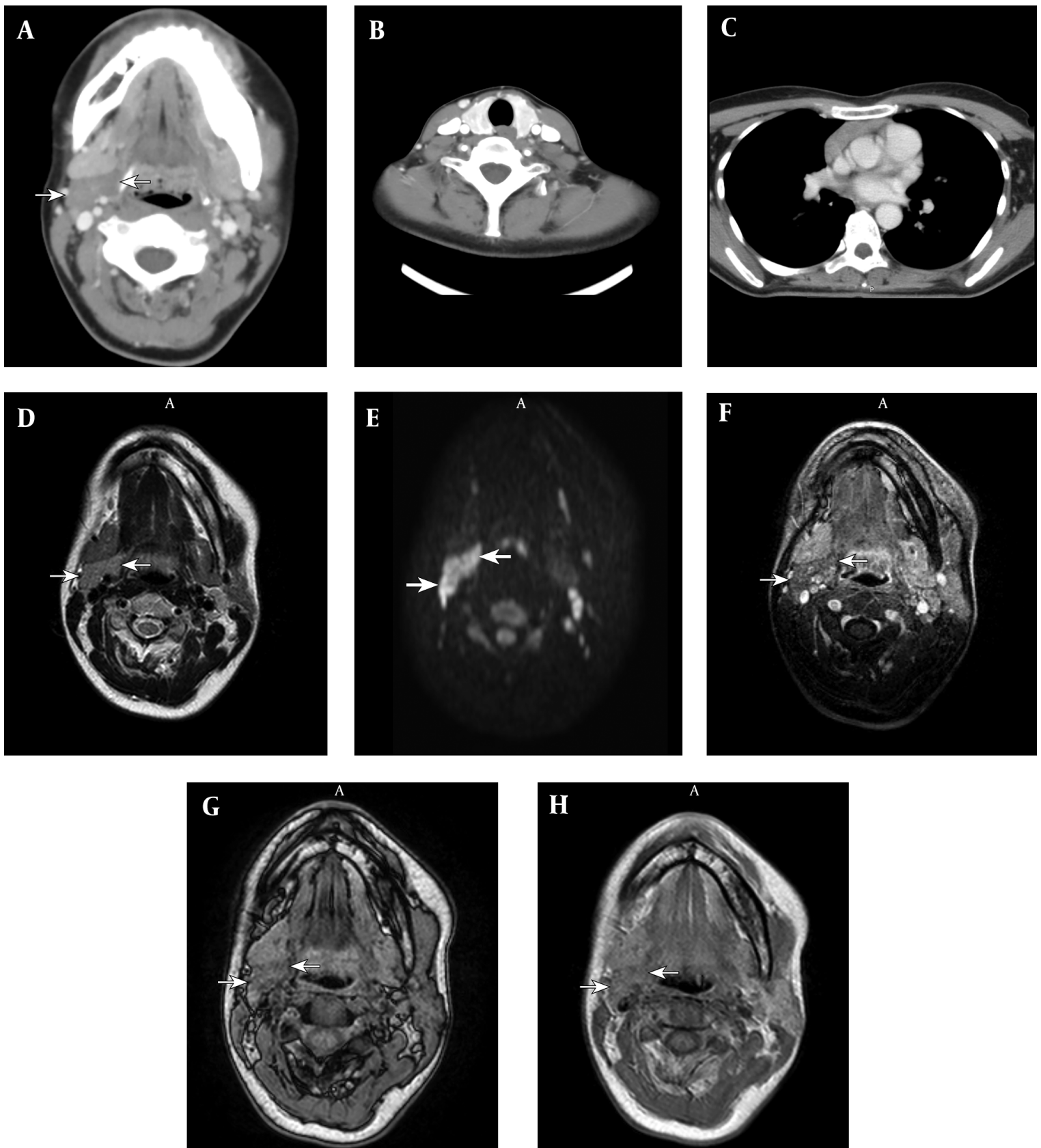

Figure 1. A 39-year-old woman with an enlarging soft mass on the right side of the neck; A, Computed tomography image shows a well-defined, homogeneous mass (arrow) between the submandibular gland and carotid artery with weak contrast enhancement; B, The thyroid gland appears heterogeneous and moderately enlarged, suggesting thyroid abnormality; C, The normal thymus appears enlarged for the patient's age; D, T2 weighted image (T2WI) of a magnetic resonance (MR) image, where the mass appears homogeneous and moderately hyperintense; E, On diffusion-weighted image (DWI), the mass appears hyperintense; F, The mass shows homogeneously weak enhancement on contrast-enhanced T1 weighted image (T1WI). Chemical shift T1 weighted image(T1WI) shows slight signal loss of the lesion on out-of-phase images (G) compared to in-phase images $(\mathrm{H})$, suggesting the presence of microscopic fat.

ening symptoms of palpitation and weight loss. On the patient's arrival to the hospital, anti-thyroid medications were not prescribed. An ultrasound examination showed a diffusely enlarged, heterogeneous hyperechoic thyroid gland with abundant vascularization. Moreover, a laboratory investigation showed an increase in both free T3 (29.3 $\mathrm{pg} / \mathrm{mL})$ and free $\mathrm{T} 4(7.75 \mathrm{ng} / \mathrm{mL})$ levels with suppressed thyroid stimulating hormone levels (TSH, $0.010 \mu \mathrm{IU} / \mathrm{mL}$ ) 
in the serum, and an increase in thyroid-stimulating immunoglobulin levels (704\%).

Fine needle aspiration (FNA) of the submandibular mass showed small, round lymphocytes without evidence of a malignant feature (Figure 2A). These cells were immunohistochemically positive for CD1a, an early T-cell marker (Figure 2B). The specimen did not contain follicular cells of the thyroid gland, and the cytomorphological and immunohistochemical findings together were suggestive of thymic tissue.

According to the above-described examinations, the submandibular mass was diagnosed as an ECT. Untreated hyperthyroidism was considered as the underlying cause of the adult-onset hyperplasia of this lesion. Thus, the patient was started on $10 \mathrm{mg}$ of oral methimazole once daily and no further surgical intervention was performed. At the 11-month follow-up, the patient was clinically asymptomatic. A laboratory examination revealed improvement in free T3 $(4.2 \mathrm{pg} / \mathrm{mL})$ and free T4 $(1.82 \mathrm{ng} / \mathrm{mL})$ serum levels, and an MR image showed marked regression of the neck mass (Figure 2C and 2D). On chemical shift T1WI, the degree of the signal difference was more noticeable than that on the previous MR image, suggesting fatty degeneration (Figure 2C and 2D). Furthermore, enlargement of the normally positioned thymus appeared improved as well. The anti-thyroid medication was continued and the patient remained asymptomatic with no change in the mass after another 2-year follow-up observation.

\section{Discussion}

The course of the disease in this patient demonstrated that hyperthyroidism could induce ECT hyperplasia even in adulthood, which can present as an enlarging mass in the neck. ECT occurs because of the complete or partial failure of thymic gland migration (1-4). Patients with an ECT usually exhibit some symptoms in their childhood, as thymus tissue undergoes significant growth during the pre-pubertal period (1-4). However, ectopic thymic tissue is rarely identified in adult patients, as they typically undergo age-related replacement by fibroadipose tissue (1-4). Thymic hyperplasia in hyperthyroidism is believed to be a benign, autoimmune process (5-7). Antibodies directed to the acetylcholine receptor play a causative role in hyperthyroidism. Moreover, the acetylcholine receptor exists in the thymus, and autoantibodies of hyperthyroidism can trigger thymic hyperplasia (5-7). Thus, we believe that this patient had an ECT that transformed to thymic hyperplasia because of uncontrolled hyperthyroidism manifesting as a clinically significant enlarging submandibular mass.

The current case also demonstrated that CT and MR imaging can contribute substantially to the diagnosis of adult-onset ECT hyperplasia. Normal thymic tissue and the hyperplastic thymus appear homogeneous with isointense or hyperintense regions when compared to muscle on T1WI and with hyperintense regions on T2WI $(4,8$, 9). On DWI, these tissues appear hyperintense because of their lymphoid component $(8,9)$, while on chemical shift MR imaging, they display a homogeneous decrease in intensity on opposed-phase images relative to that on in-phase images, as these tissues typically contain microscopic fat (10). In the case described in the current report, the submandibular mass appeared homogeneous, with a well-defined contour and no calcification or necrotic portions. Radiological characteristics of the mass matched the above mentioned imaging findings of thymic tissue. Moreover, normally positioned thymus enlargement was suggestive of adult-onset ECT hyperplasia. Therefore, MR imaging - especially chemical shift T1WI - was helpful in distinguishing ECT hyperplasia from other malignant entities.

A definitive diagnosis of ECT usually requires open incisional or excisional biopsy; however, such procedures increase the risk of surgical and anesthetic complications (3, 4). A previous report demonstrated that FNA with a cytometric analysis showing a lymphocyte population of almost entirely immature T cells that coexpress CD4 and CD8 and show positivity for the early T-cell markers, CD1a and TdT, can also lead to an appropriate ECT diagnosis (3). In this case, we performed immunohistochemical staining with CD1a submitted on the FNA specimen instead of flow cytometry. Together, cytomorphological and immunohistochemical findings of the FNA specimen supported the diagnosis of ECT hyperplasia.

The current case revealed marked regression of ECT hyperplasia after appropriate treatment of the patient's hyperthyroidism; thus, unnecessary surgical intervention was avoided. Previous reports have indicated that thymic hyperplasia associated with hyperthyroidism can regress after anti-thyroid treatment (5-7). Furthermore, it should be noted that there is no known increased risk of malignancy associated with thymic hyperplasia or ECT (57). Following our findings, we presented the two following options to the patient: 1) Close follow-up with antithyroid treatment, 2) Further intervention, such as excisional biopsy or surgery, to reach a definitive diagnosis. The patient selected the former option. Indeed, following treatment with oral methimazole, the lesion showed marked decrease in size with fatty degeneration when thyroid function was normalized. No recurrent enlargement of the mass was observed.

To the best of our knowledge, this is the first case of hyperthyroidism-induced ECT hyperplasia showing obvious clinical manifestation in adulthood. However, there 



Figure 2. A, Fine needle aspiration (FNA) of the mass shows small, round lymphocytes without evidence of malignant features (original magnification $500 \times$ ); B, These cells were immunohistochemically positive for CD1a, an early T-cell marker (original magnification $500 \times$ ); C-D, Follow-up magnetic resonance imaging (MRI) shows marked regression of the mass. On out-of-phase T1 weighted image (T1WI), the mass shows obvious signal loss compared to the in-phase T1WI. The degree of signal difference was more noticeable than that in the previous MR image, suggesting fatty degeneration.

may be some cases which remain unrecognized because they are subclinical, or because of spontaneous regression after anti-thyroid treatment. Therefore, ECT hyperplasia must be considered in the differential diagnosis of an enlarging neck mass in adult patients with uncontrolled hyperthyroidism.

In conclusion, the current report revealed that ECT hyperplasia could be induced by hyperthyroidism in adult- hood, and could present as an enlarging submandibular mass. In such cases, imaging tools, especially CT and MR techniques can be of help in therapeutic diagnosis. Finally, as shown by the current case, the lesion may regress if the underlying hyperthyroidism is appropriately treated, thus avoiding further surgical intervention. 


\section{Acknowledgments}

The authors are grateful to the translational research and resource core (Tsukuba Human Tissue Diagnostic Center, University of Tsukuba Hospital) for their skillful technical assistance in immunohistochemical staining.

\section{Footnotes}

Authors' Contributions: None declared.

Financial Disclosure: The authors have no conflict of interest.

Funding/Support: None declared.

\section{References}

1. Kotani H, Ishida T, Miyao M, Manabe S, Kawai C, Abiru H, et al. Ectopic cervical thymus: a clinicopathological study of consecutive, unselected infant autopsies. Int J Pediatr Otorhinolaryngol. 2014;78(11):191722. doi: 10.1016/j.ijporl.2014.08.024. [PubMed: 25213423].

2. Tabatabaie SA, Hashemi SM, Sanei B, Sanei MH. The frequency of ectopic thymic tissue in the necks of patients without any thymic disease. Med Sci Monit. 2007;13(6):CR283-5. [PubMed: 17534235].
3. Tunkel DE, Erozan YS, Weir EG. Ectopic cervical thymic tissue: diagnosis by fine needle aspiration. Arch Pathol Lab Med. 2001;125(2):27881. doi: 10.1043/0003-9985(2001)125<0278:ECTT>2.0.CO;2. [PubMed: 11175652].

4. Zielke AM, Swischuk LE, Hernandez JA. Ectopic cervical thymic tissue: can imaging obviate biopsy and surgical removal?. Pediatr Radiol. 2007;37(11):1174-7. doi: 10.1007/s00247-007-0598-7. [PubMed: 17851657].

5. Murakami M, Hosoi Y, Negishi T, Kamiya Y, Miyashita K, Yamada $\mathrm{M}$, et al. Thymic hyperplasia in patients with Graves' disease. Identification of thyrotropin receptors in human thymus. J Clin Invest. 1996;98(10):2228-34. doi: 10.1172/JCI119032. [PubMed: 8941638].

6. Suh H, Rosen JE, Doherty GM, Saunders BD, McAneny D. Graves' disease and thymic hyperplasia. Surgery. 2013;154(6):1473-7. doi: 10.1016/j.surg.2013.09.004. [PubMed: 24238061].

7. Takami K, Omiya H, Higashiyama M, Maeda J, Okami J, Oda K, et al. A case report of large thymic hyperplasia associated with hyperthyroidism. Ann Thorac Cardiovasc Surg. 2009;15(6):404-7. [PubMed: 20081752].

8. Nasseri F, Eftekhari F. Clinical and radiologic review of the normal and abnormal thymus: pearls and pitfalls. Radiographics. 2010;30(2):413-28. doi: 10.1148/rg.302095131. [PubMed: 20228326].

9. Nishino M, Ashiku SK, Kocher ON, Thurer RL, Boiselle PM, Hatabu H. The thymus: a comprehensive review. Radiographics. 2006;26(2):33548. doi: 10.1148/rg.262045213. [PubMed: 16549602].

10. Takahashi K, Inaoka T, Murakami N, Hirota H, Iwata K, Nagasawa K, et al. Characterization of the normal and hyperplastic thymus on chemical-shift MR imaging. AJR Am J Roentgenol. 2003;180(5):1265-9. doi: 10.2214/ajr.180.5.1801265. [PubMed: 12704035]. 